Check for updates

Cite this: RSC Adv., 2019, 9, 33722

Received 9th June 2019

Accepted 4th September 2019

DOI: $10.1039 / c 9 r a 04253 a$

rsc.li/rsc-advances

\section{Preparation of an electrospun tubular PU/GE nanofiber membrane for high flux oil/water separation}

\begin{abstract}
Yan-jie Wu, Chang-fa Xiao (D) * and Jian Zhao
A simple, tubular structure polyurethane/graphene (PU/GE) nanofiber membrane for continuous oil/water separation was prepared using the following strategies: a polyester (PET) fiber braided tube was used for reinforcement, stearic acid (SA) was used to assist GE dispersion, and a PU solution containing GE was used to cover the outer layer of the PET fiber braided tube using the electrospinning method. Specifically, the PU/GE nanofiber membrane has a multi-branched structure. The tubular braid reinforced (TBR) PU/GE nanofiber membrane was characterized using field emission scanning electron microscopy (FESEM), transmission electron microscopy (TEM), confocal scanning microscopy (CSM) and capillary flow porometry. The contact angle results showed that the TBR PU/GE nanofiber membrane had good hydrophobic and lipophilic properties. The obtained membranes had good oil/water selectivity for oil-water separation (with a separation efficiency up to 99\%). In addition, the optimized membrane can be effectively employed to separate a surfactant-stabilized water-in-oil emulsion with a separation efficiency up to $90 \%$ and a high permeate flux $\left(137.5 \mathrm{~L} \mathrm{~m}^{-2} \mathrm{~h}^{-1}\right)$. Our TBR PU/GE nanofiber membrane is therefore a desirable material for the highly efficient separation of water-in-oil emulsions, and shows broad application prospects in the field of oil/water separation.
\end{abstract}

\section{Introduction}

Oil inevitably leaks into the water during the processes of oil exploration, refining, transportation and use. Oily wastewater poses a serious threat to the ecological environment and human health. ${ }^{1,2}$ The separation of oil/water mixtures, especially surfacestable water-in-oil emulsions, has become a worldwide challenge. Traditional oil-water separation technologies, such as gravity separation, skimming and flotation are not only inefficient, but also have high operating costs. ${ }^{3-5}$ Moreover, some of them are not suitable for the separation of water-in-oil emulsions. Recently, membrane technology has been widely studied owing to its high degree of separation, high efficiency, good selectivity and different modes of separation. ${ }^{6}$ Two rules should be considered for the separation of an oil-water emulsion: ${ }^{7-10}$ (i) the selective wettability of water (or oil) should provide the driving force for separation based on the hydrophobicity and lipophilicity; and (ii) the connected pores with an appropriate pore size should provide channels for filtrate infiltration.

Nanofiber membranes, which usually have a diameter of tens or hundreds of nanometers, possess interconnected pores, a relatively uniform pore size distribution and high porosity.

School of Textiles, State Key Laboratory of Separation Membranes and Membrane Processes, Tianjin Polytechnic University, No. 399 Binshui West Street, Xiqing District, Tianjin 300387, P. R. China. E-mail: cfxiao@tjpu.edu.cn; Tel: +86 22 83955299
This reduces the operating pressure of the nanofiber membrane but increases the permeate flux..$^{\mathbf{1 1}-13}$ Nanofibers can be prepared by various methods including traction, template self-assembly and electrospinning. ${ }^{\mathbf{1 4 - 1 6}}$ Among these methods, electrospinning technology is considered to be a reliable spinning technology for fabricating nanofibers or porous membranes owing to its relative simplicity, low energy consumption, and wide source of raw materials such as polyethersulfone (PES), ${ }^{17}$ polysulfone (PS), ${ }^{18}$ polyvinylidene divinyl ether fluorine (PVDF), ${ }^{19}$ polyurethane (PU), ${ }^{20}$ polyacrylonitrile (PAN), ${ }^{21}$ and so forth. Electrospun nanofiber membranes composed of continuously entangled fibers possess a three-dimensional (3D) network structure, which is suitable for a variety of applications, including filtration and desalting. ${ }^{22-24}$

Polyurethane with unique characteristics including biodegradability, biocompatibility and low cost has become increasingly desirable owing to the environmental and economic demands. Although previous work has proved the feasibility of preparing PU nanofibers by electrospinning, it need to increase the specific area of the membrane by designing different shapes. Hollow fiber membranes have an important and unique form of membrane configuration owing to their high specific surface area, high packing density and self-supporting nature. In membrane separation applications they have a significant advantage for solving serious water shortages, environmental pollution and energy shortages. ${ }^{25,26}$ 
In this paper, a tubular braid reinforced (TBR) polyurethane/ graphene (PU/GE) nanofiber membrane was fabricated by electrospinning using a PET fiber braided tube as a reinforcement and a casting solution of PU containing GE as the spinning solution. The effects of different GE contents on the morphology, porosity, pore size distribution and contact angle of the TBR PU/GE nanofiber membrane were investigated. The separation performance of the as-prepared TBR PU/GE nanofiber membrane on an oil/water mixture and a water-in-oil emulsion was also studied.

\section{Experimental}

\subsection{Materials}

Polyurethane (285, fiber grade, density $=1.20 \mathrm{~g} \mathrm{~cm}^{-3}$ ) resin was purchased from Bayer, Germany. Graphene (GE, KNGG5) with a thickness of less than $5 \mathrm{~nm}$, and a flake size of 0.1-5 $\mu \mathrm{m}$ was purchased from Xiamen Kaina Graphene Technology Co., Ltd. (Xiamen, China). N,N-Dimethyl formamide (DMF, analytical reagent, 98\%), stearic acid (SA, analytical reagent), sodium chloride and Span 80 were purchased from Tianjin Kemiou Chemical Reagent Co., Ltd. The PET braided tube was supplied by Tianjin Boanxin Co., Ltd. (Tianjin, China). The PET braided

Table 1 The composition of the PU/GE doping solution

\begin{tabular}{llllll}
\hline Sample & PU (wt\%) & NaCl (wt\%) & SA (wt\%) & DMF (wt\%) & GE (wt\%) \\
\hline PU/GE4-0 & 16 & 0.2 & 4 & 79.8 & 0 \\
PU/GE4-0.1 & 16 & 0.2 & 4 & 79.7 & 0.1 \\
PU/GE4-0.3 & 16 & 0.2 & 4 & 79.5 & 0.3 \\
PU/GE4-0.5 & 16 & 0.2 & 4 & 79.3 & 0.5
\end{tabular}

tube outer diameter was $1.8 \mathrm{~cm}$, with 48 spindles, and weighed 1.55 grams per meter. Kerosene was obtained from Tianjin Kailida Chemical Co., Ltd (Tianjin, China). All chemicals were of analytical grade, and used without further purification.

\subsection{Preparation of the TBR PU/GE nanofiber membrane}

The TBR PU/GE nanofiber membrane was prepared using the electrospinning method. $0.2 \mathrm{wt} \% \mathrm{NaCl}, 4 \mathrm{wt} \% \mathrm{SA}$ and different contents of GE were firstly added into a DMF solution under ultrasonic treatment for $1 \mathrm{~h}$. The solution was placed in a $70{ }^{\circ} \mathrm{C}$ water bath under mechanical stirring, followed by the addition of $16 \mathrm{wt} \% \mathrm{PU}$ resin and continuous stirring to form a uniform solution. After the above mentioned uniform solution was placed in a vacuum oven for $1 \mathrm{~h}$ to eliminate the air bubbles, a PU/GE doping solution for electrospinning was obtained. The composition of the PU/GE electrospinning solution is listed in Table 1.

The TBR PU/GE nanofiber membrane was produced using a TL-Pro (Shenzhen Tongli Weina Technology Co., Ltd.) type electrospinning apparatus. The spinning process parameters were set as follows: the positive pressure of the spinneret was $25.5 \mathrm{kV}$, the negative pressure of the receiving device was -5.5 $\mathrm{kV}$, the receiving distance was $10 \mathrm{~cm}$, the receiving device speed was $1500 \mathrm{rpm}$, the spinning solution injection speed was $2.1 \mathrm{~mL}$ $\mathrm{h}^{-1}$, and the spinning temperature and the relative humidity was $25^{\circ} \mathrm{C}$ and $50 \pm 5 \%$, respectively. Note that this differs from the ordinary electrospinning process as the receiving body was a PET fiber braided tube during the spinning process. Here, the PET fiber braided tube was used as a reinforcement to improve the mechanical properties of the TBR PU/GE nanofiber membrane. The preparation process for the TBR PU/GE nanofiber membrane is shown in Fig. 1.

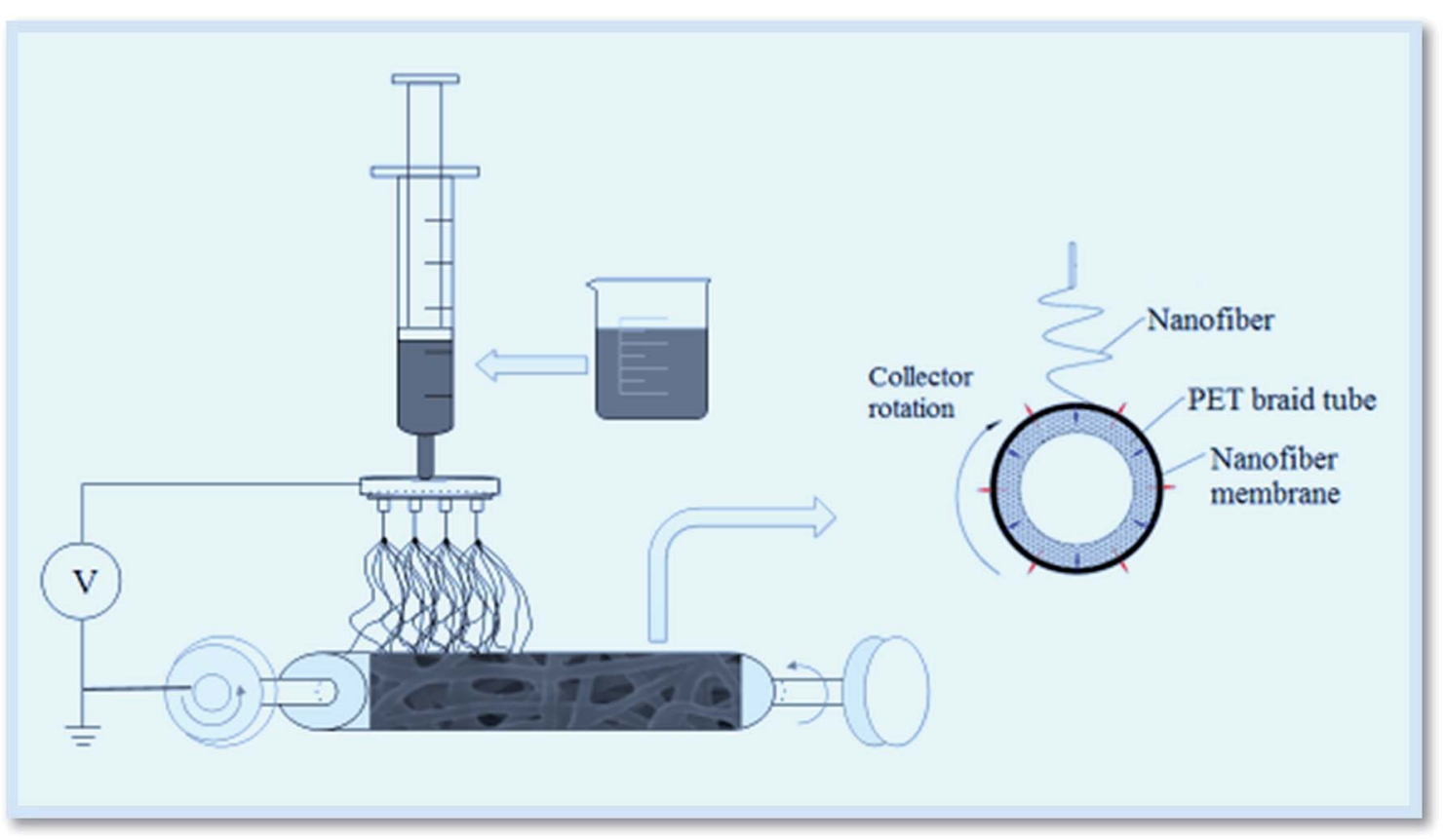

Fig. 1 The preparation process of the TBR PU/GE nanofiber membrane. 


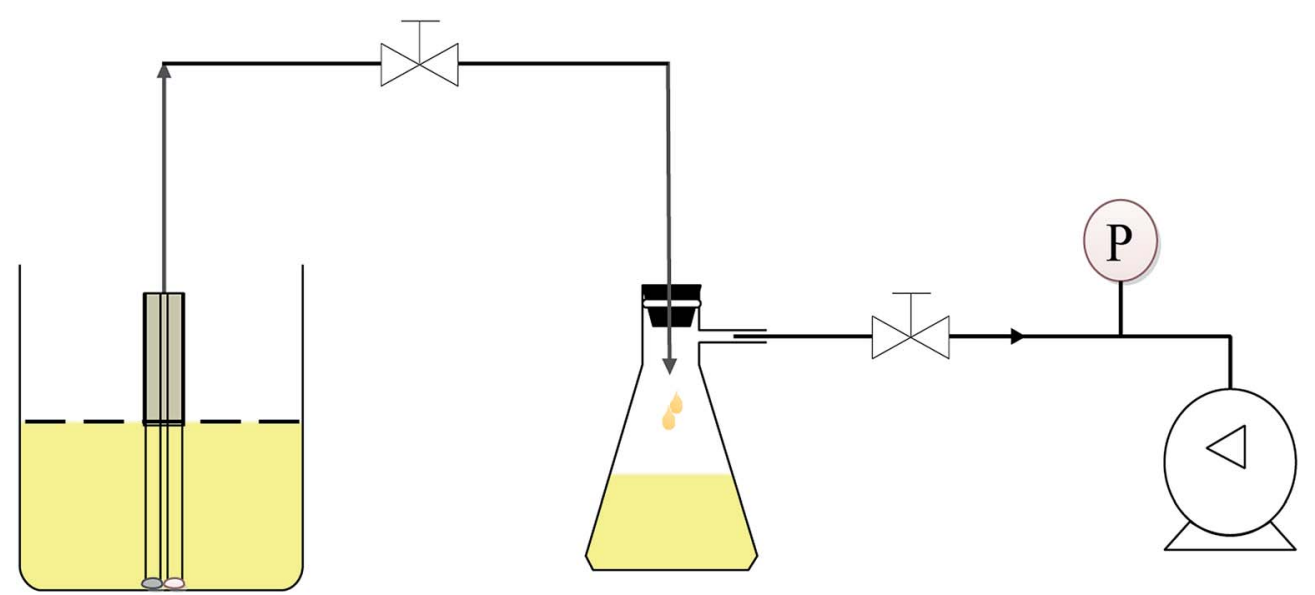

Fig. 2 The flux tester for the TBR PU/GE nanofiber membrane.
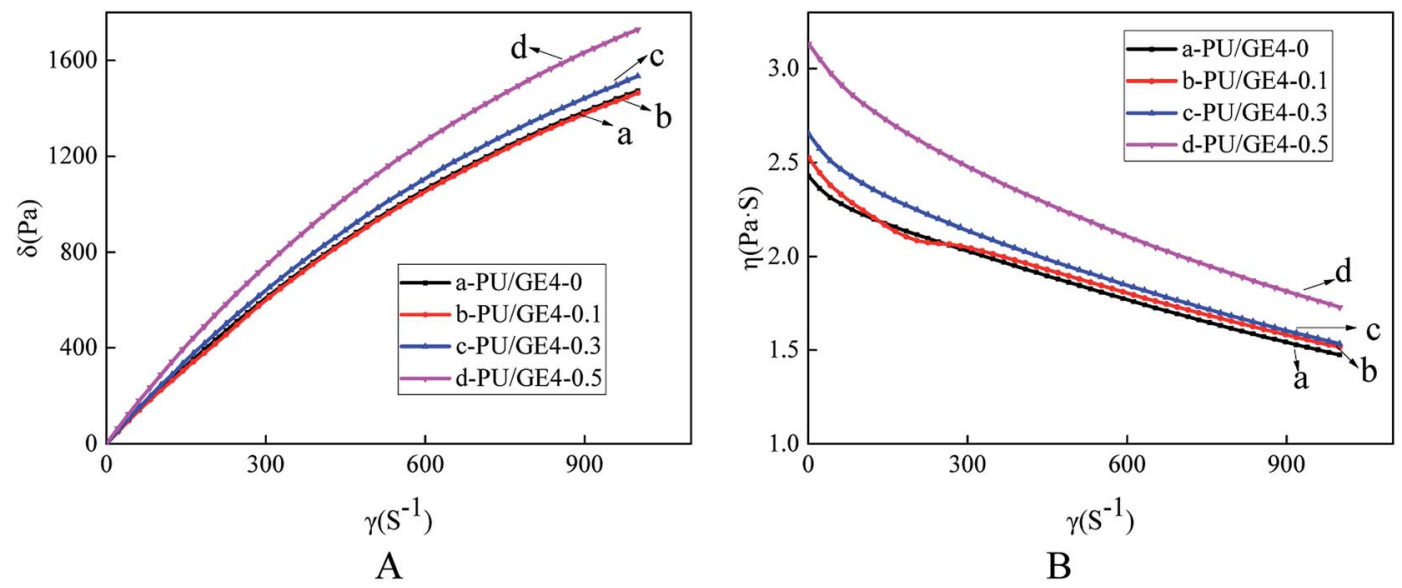

Fig. 3 Effect of GE content on the $\sigma-\gamma$ curve (A) and $\eta-\gamma$ curve (B).

The cross-section structure of the TBR nanofiber membrane is clearly shown in Fig. 1. During the electrospinning process, the self-supporting tension of the PET fiber braided tube and the inward winding tension of the nanofiber membrane allowed the nanofiber membrane to form a close combination. Moreover, the surface of the PET fiber braided tube was relatively rough, which promoted a better interfacial bonding performance between them.

\subsection{Water-in-oil emulsion preparation}

A water-in-oil emulsion containing an emulsifier was prepared by high speed stirring. Firstly, surban 80, as a water-in-oil emulsifier ( $\mathrm{HLB}=4.3 ; 0.2 \mathrm{~g}$ ) was added to $100 \mathrm{~mL}$ kerosene under high speed stirring for $1 \mathrm{~h}$. Then, $100 \mu \mathrm{L}$ of water was added to the mixture, and the mixture was stirred at a high speed for $0.5 \mathrm{~h}$. The above described operation was repeated ten times. Finally, the mixture was stirred at high speed for a further $3 \mathrm{~h}$ to obtain a surfactant-stabilized water-in-oil emulsion.

\subsection{Membrane characterization}

The rheological behavior of the doping solutions was investigated with a rheometer (HAAKE MARS, Thermo Fisher
Scientific). The morphology of the TBR PU/GE nanofiber membrane was observed using field emission scanning electron microscopy (FESEM, Nova Nano 230, Netherlands FEI), confocal scanning microscopy (CSM, Zeiss CSM 700, Germany) and transmission electron microscopy (TEM, H7650, Hitachi, Japan). The pore size and the distribution of the as-prepared membrane was determined using capillary flow porometry (POROLUX1000, Belgium). The membrane porosity was determined using the gravimetric method. ${ }^{27,28}$ The static contact angle was measured using a contact angle goniometer (DSA 100, KRÜSS, Germany). The mechanical properties of the TBR PU/GE nanofiber membrane were tested using an electronic stretching machine (M350-5Kn, SDLATLAS, British) at room temperature.

Table 2 Non-Newtonian index of the PU/GE doping solution with different GE contents $\left(25^{\circ} \mathrm{C}\right)$

$\begin{array}{lllll}\text { Polymer solution } & \text { PU/GE4-0 } & \text { PU/GE4-0.1 } & \text { PU/GE4-0.3 } & \text { PU/GE4-0.5 } \\ \text { Non-Newtonian } & 0.9051 & 0.9012 & 0.8969 & 0.8866 \\ \text { index } & & & & \end{array}$




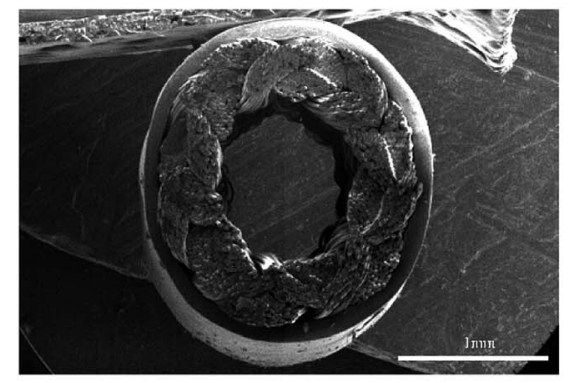

(A1)

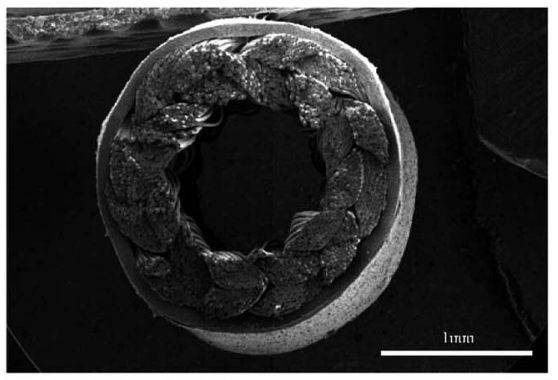

(B1)

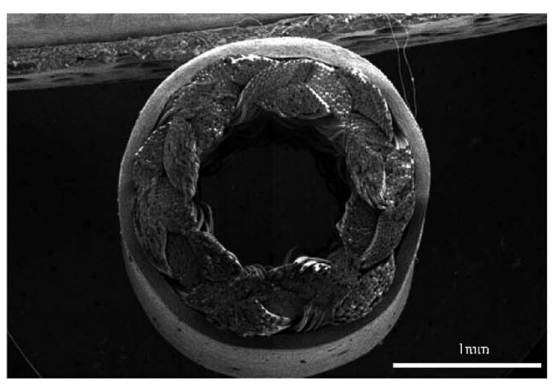

(C1)

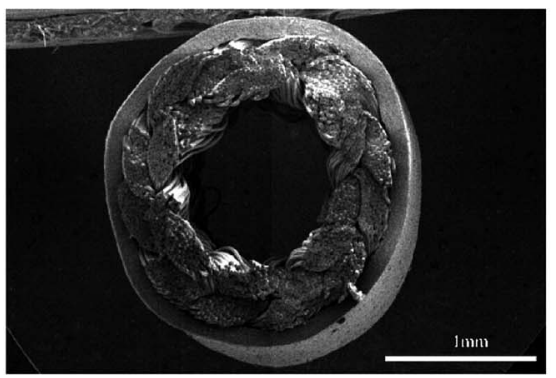

(D1)

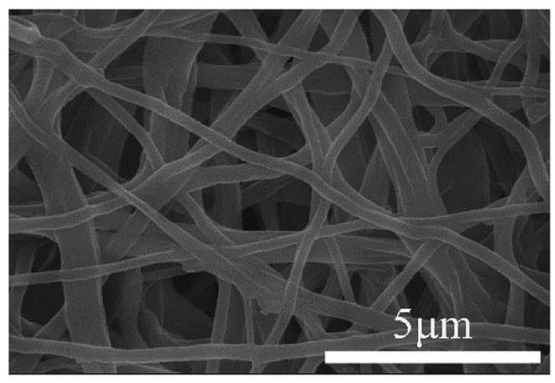

(A2)

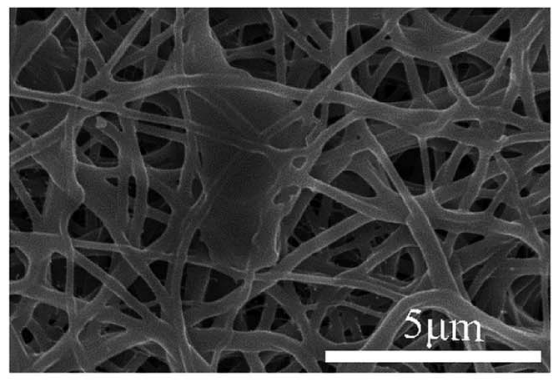

(B2)

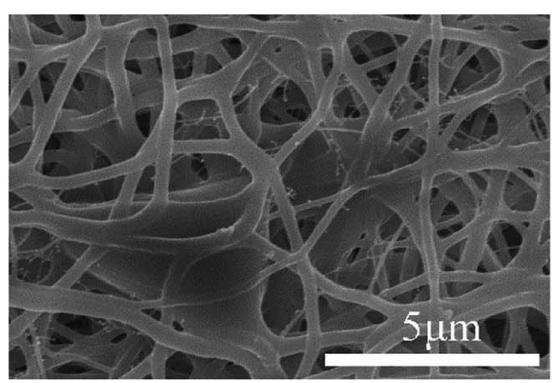

(C2)

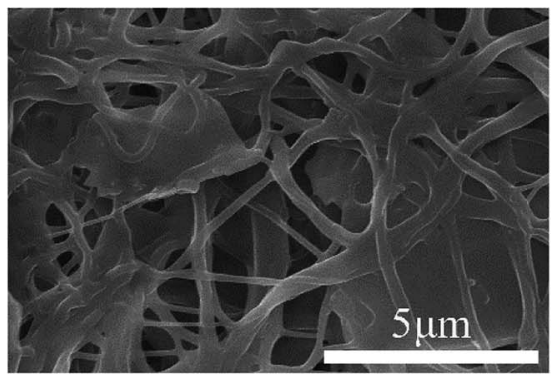

(D2)

Fig. 4 The morphology of the TBR PU/GE nanofiber membrane. (A) PU/GE4-0; (B) PU/GE4-0.1; (C) PU/GE4-0.3; and (D) PU/GE4-0.5; (1) cross section; and (2) surface.

\subsection{Separation performance test}

The prepared TBR PU/GE nanofiber membrane was placed in a vacuum filtration apparatus. ${ }^{29}$ The continuous oil removal from the water surface and surfactant-stabilized water-in-oil emulsion via the TBR PU/GE nanofiber membrane was measured using a laboratory scale experimental setup with -0.5 bar, as shown in Fig. 2. The permeation flux was calculated according to the following formula:

$$
J=\frac{V}{A \times t}
$$


In which $J$ is the permeation flux $\left(\mathrm{L} \mathrm{m}^{-2} \mathrm{~h}^{-1}\right), V$ is the volume of permeation (L), $A$ is the effective area of the membrane $\left(\mathrm{m}^{2}\right)$, and $t$ is the testing time (h).

An optical microscopy image of the emulsion and filtrate was obtained using optical microscopy (Olympus BX43, Olympus). The moisture content of the filtrate was measured using a Karl Fischer moisture meter (Byes2000, Byes). The separation efficiency of the membrane was calculated using the following equation:

$$
R=\left(1-\frac{C_{\mathrm{P}}}{C_{\mathrm{f}}}\right) \times 100 \%
$$

In which $R$ is the separation efficiency, and $C_{\mathrm{f}}$ and $C_{\mathrm{p}}$ are the concentration in the feed and the permeate solution, respectively.

\section{Results and discussion}

\subsection{Rheological properties of the doping solution}

The effect of the GE content on the rheological behavior of the doping solution is shown in Fig. 3. At the same shear rate $(\gamma)$, it can be seen that the shear stress of the doping solution without GE was smaller than the shear stress of the doping solution with the addition of GE. Furthermore, as the GE content increased, the shear stress and the viscosity $(\eta)$ of the doping solution also increased. Note that GE had a significant thickening effect and enhanced mechanical properties of the membrane are achieved. To further study the effect of the GE content on the nonNewtonian behavior of doping solutions, the slope of the log shear stress $(\log \sigma)$ versus the $\log$ shear rate $(\log \gamma)$ was described using the non-Newtonian index $(n)$ according to the definition, as shown in Table 2 . When $n<1$, the fluid was a shear thinning fluid. With the addition of GE, the nonNewtonian index $n$ of the doping solutions decreased. In summary, the doping solution exhibited a good fluidity.

\subsection{Morphology}

The morphology of the TBR PU/GE nanofiber membrane is shown in Fig. 4. Obviously, the cross-section consists of a PET

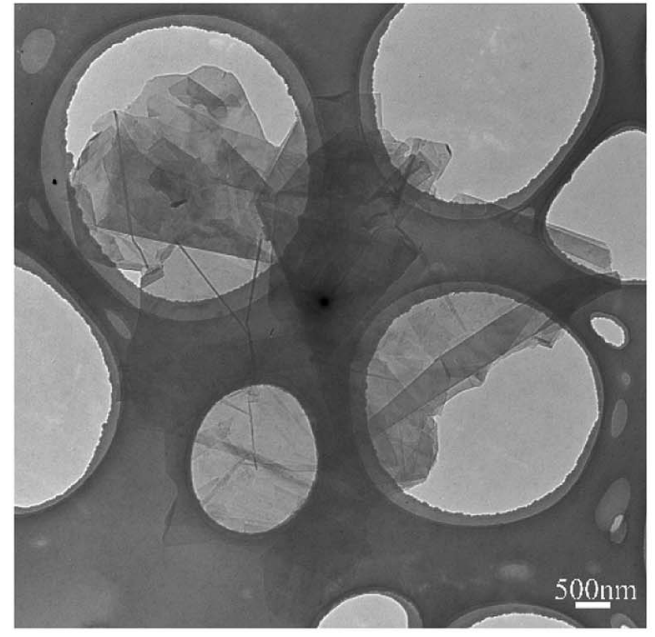

(A)

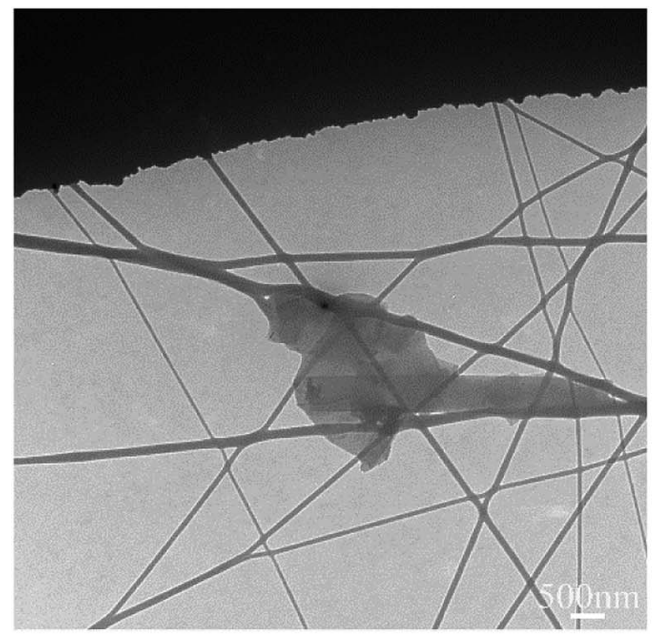

(C)

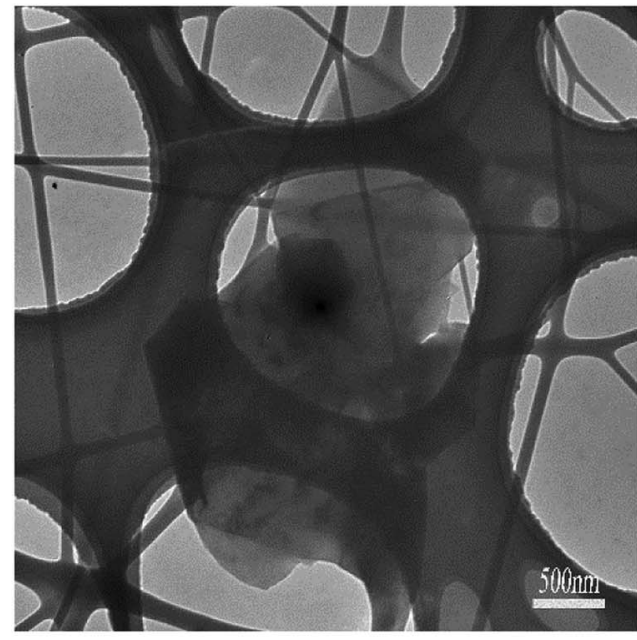

(B)

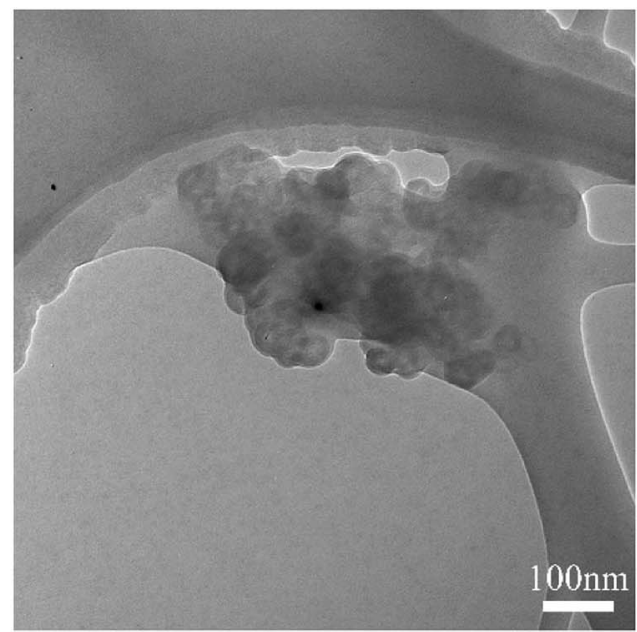

(D)

Fig. 5 The TEM morphology of various samples: (A) GE; (B) PU/GE4-0.1; (C) PU/GE4-0.3; and (D) PU/GE4-0.5. 
fiber braided tube and a PU/GE nanofiber membrane, and the nanofiber membrane was tightly wrapped around the PET fiber braided tube. This ensured that there was a robust bonding performance between the PET fiber braided tube and the nanofiber membrane, which imparted excellent mechanical properties to the reinforced tubular nanofiber membrane. It can be seen from Fig. 4A2-C2 that the surface of the nanofiber membrane was a relatively smooth, continuous structure and not a beaded structure, indicating that the parameters used in the electrospinning process were reasonable. With the addition of GE, GE nanosheets were observed radially on the surface of the TBR PU/GE nanofiber membrane. Owing to the large size of GE, GE was exposed outside the nanofiber. In addition, it can be seen that GE was intimately attached to the nanofiber along the axial direction. This structure can not only increase the efficiency because of the large specific surface area produced by GE, but also ensure that the GE and nanofiber are tightly combined. The maximum contact area of the GE and nanofiber prevent GE fall-off from the nanofiber during the application. With the increase of the GE content, the TBR PU/GE nanofiber membrane $(0.3 \mathrm{wt} \%)$ presented a tree-like structure. The thicker nanofiber acted as a support for the GE and multi-level nanofibers like a tree trunk, and the thinner fiber formed by splitting was like a tree branch. The results could be attributed to the increased conductivity. ${ }^{30}$ The diameter of the nanofiber became smaller and the dimensional uniformity decreased. With a further increase of the GE content, agglomeration of the GE sheet may have occurred.

The TEM morphology of the TBR PU/GE nanofiber membrane with different GE contents is shown in Fig. 5. Fig. 5a shows the
TEM morphology of the GE nanosheet, which shows a typical pleated morphology with a large size and multilayer structure. The TEM morphology of the TBR PU/GE nanofiber membrane showed that GE was embedded in the PU nanofiber and distributed along the axial direction of the nanofiber, indicating that the GE was successfully doped into the PU/GE nanofiber membrane (Fig. 5b, $c$ and d). The GE in the TBR PU/GE nanofiber membrane had the same typical pleated morphology, but the morphology of the GE transformed from a stretched nanosheet to become agglomerated, indicating that the thickness of the GE in the TBR PU/GE nanofiber membrane was slightly thicker. When the content of GE exceeded $0.3 \mathrm{wt} \%$, the aggregation of GE occurred in the TBR PU/GE nanofiber membrane.

The 3D surface roughness image of the PU/GE nanofiber membrane obtained using CSM apparatus is shown in Fig. 6. It can be seen that the roughness of the PU nanofiber membrane was small. The average roughness values $\left(R_{\mathrm{a}}\right)$ of the PU/GE4-0, PU/GE4-0.1, PU/GE4-0.3, and PU/GE4-0.5 nanofiber membranes were $1.252,2.289,2.604$, and $3.590 \mu \mathrm{m}$, respectively. With the increased GE content, the TBR PU/GE nanofiber membrane presented an obvious tree-like structure, therefore the surface roughness of the PU/GE nanofiber membrane was gradually increased, which was favorable for the hydrophobic and lipophilic properties of membrane and application for oil/ water separation. The change in the roughness value is consistent with the change in the morphology.

\subsection{Porosity and pore size distribution}

The porosity of the TBR PU/GE nanofiber membranes with different GE contents is shown in Fig. 7. The porosity of the TBR

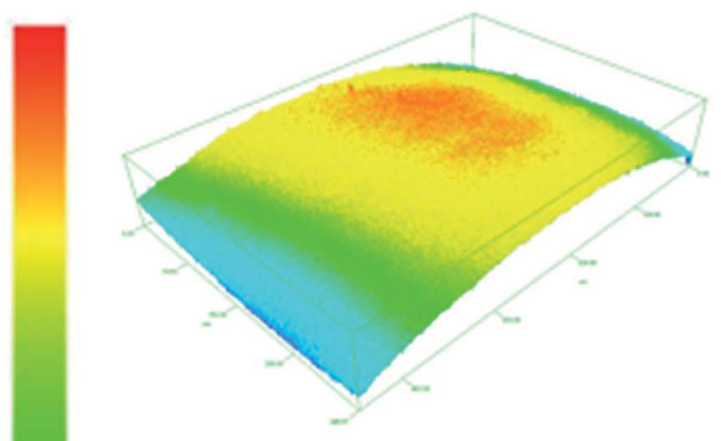

A

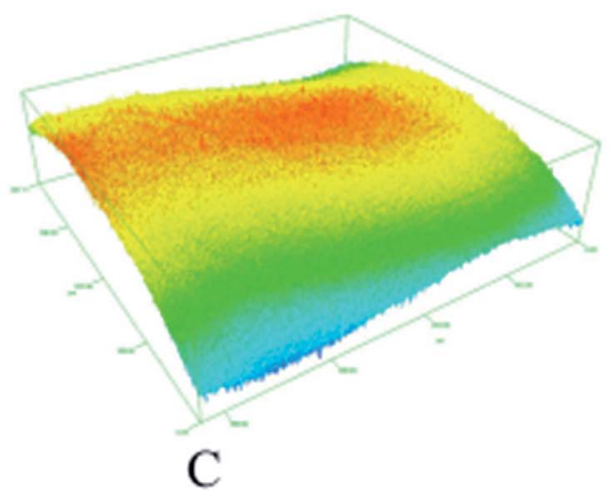

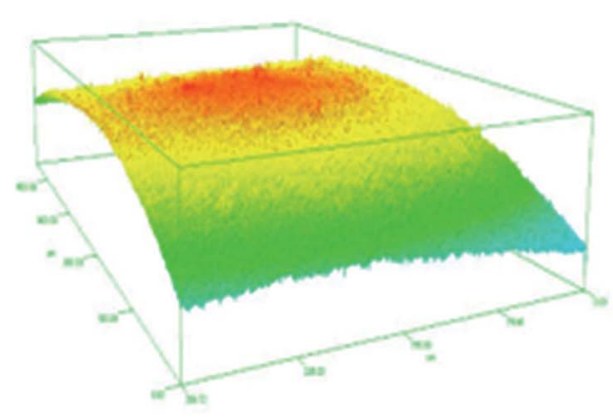

B

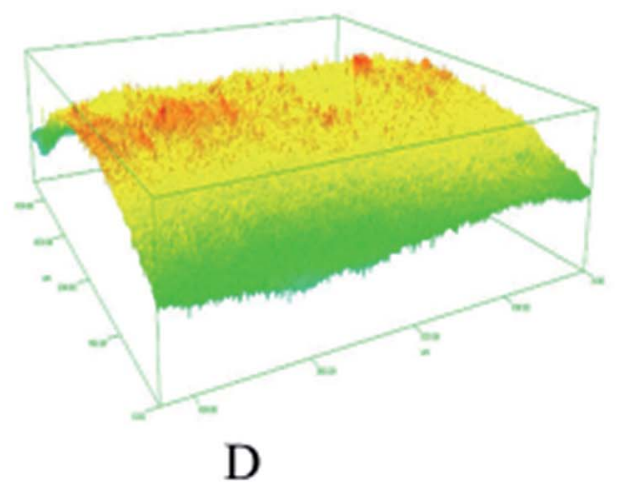

Fig. 6 CSM images of the TBR PU/GE nanofiber membranes. (A) PU/GE4-0; (B) PU/GE4-0.1; (C) PU/GE4-0.3; and (D) PU/GE4-0.5. 


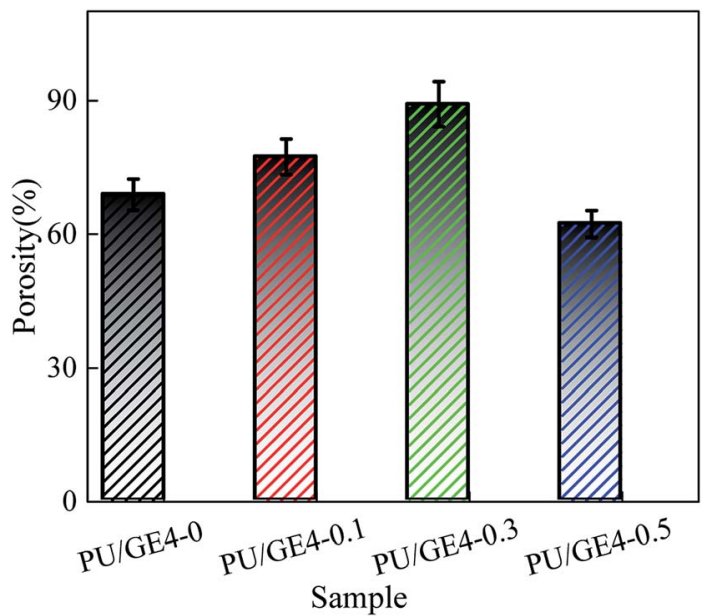

Fig. 7 The porosity of the TBR PU/GE nanofiber membrane.

PU/GE nanofiber membrane was as high as $60 \%$, which was contributed to by the $3 \mathrm{D}$ network porous structure. When the GE content increased from 0 to $0.5 \mathrm{wt} \%$, the porosity of the PU/ GE nanofiber membrane first increased and then decreased. This may be due to the introduction of GE, the finer diameter of the nanofibers, and the increased porosity of the nanofiber membrane. However, the excessive GE increased the viscosity of the doping solution, leading to a reduced number of pores and the pore size owing to GE agglomeration.

As can be seen from Fig. 8, the pore size distribution of the TBR PU/GE4-0 nanofiber membrane was wide. The average pore diameter of the PU/GE4-0.1, PU/GE4-0.3 and PU/GE4-0.5 nanofiber membrane was $0.19,0.48$, and $0.07 \mu \mathrm{m}$, respectively. With the increased GE content, the average pore size first decreased and then increased, but the pore size distribution was narrow. The results suggested that the porosity and pore size distribution could be controlled by adjusting the GE content.

\subsection{Mechanical properties}

The TBR nanofiber membrane can greatly improve the mechanical properties of the composite membrane, and the testing results are shown in Fig. 9. It can be seen that the breakage strength of the PU/GE nanofiber membrane without the PET braided tube was only about $10 \mathrm{MPa}$. While the breakage strength of the PET braided tube reinforced PU/GE nanofiber membrane was about $500 \mathrm{MPa}$, which was as high as 50 times that of the pristine PU/GE nanofiber membrane. In addition, it can be seen that the breakage strength of the TBR
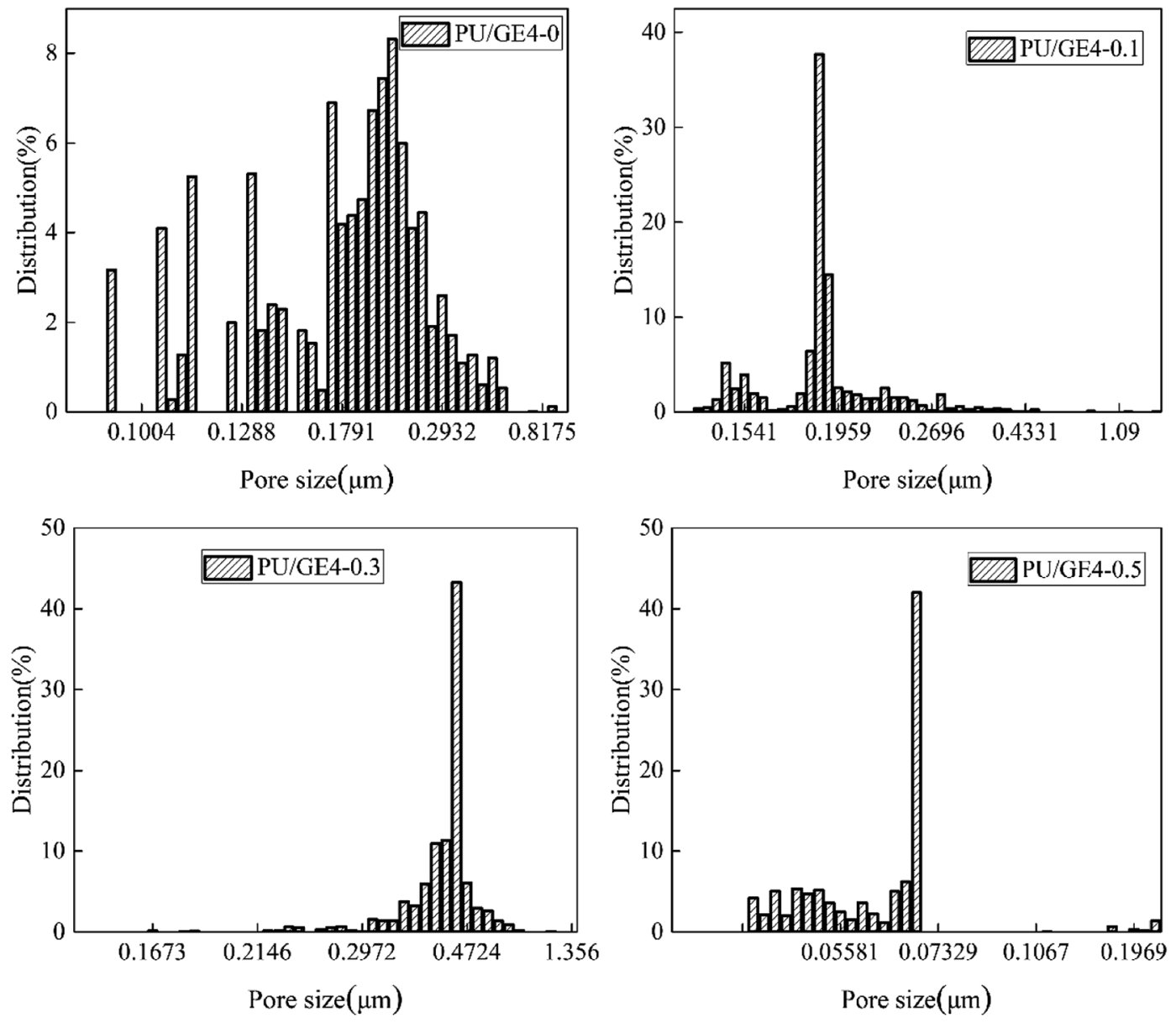

Fig. 8 The pore size distribution of the TBR PU/GE nanofiber membrane. 


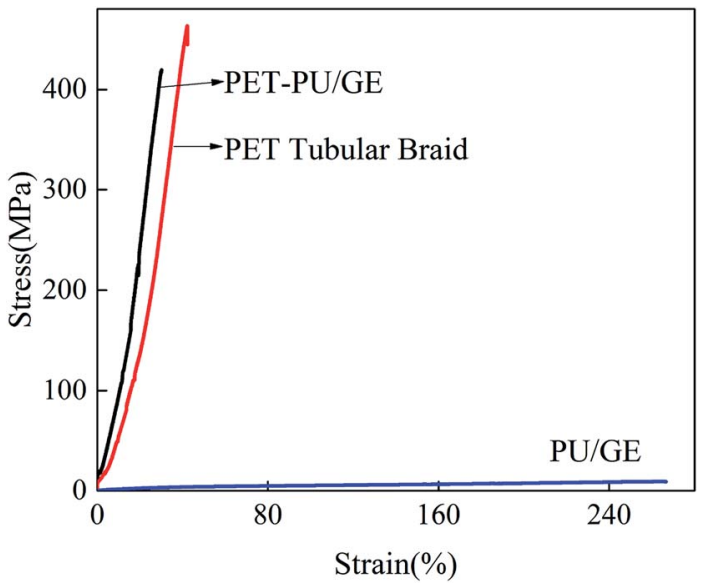

Fig. 9 The mechanical properties of the TBR PU/GE nanofiber membrane.

PU/GE nanofiber membrane was close to that of the PET braided tube as the mechanical properties of the TBR PU/GE nanofiber membrane were mainly provided by the PET fiber

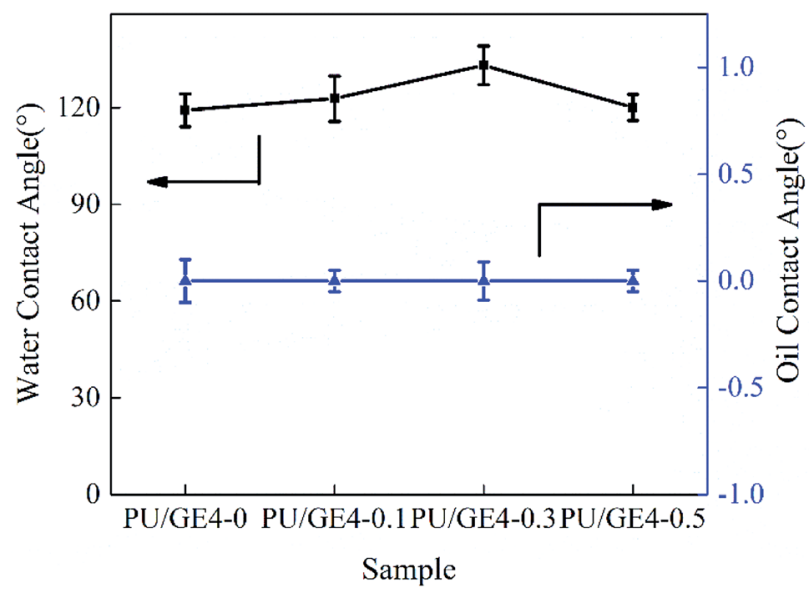

Fig. 10 Contact angles for the TBR PU/GE nanofiber membrane. braided tube. From Fig. 9 it can be seen that the breaking elongation of the PU/GE nanofiber membrane without the PET braided tube was approximately $240 \%$, while the breaking elongation of the TBR PU/GE nanofiber membrane became lower. This can be explained by the fact that the PET braided tube limits the variation of the nanofiber membrane along the length direction. Moreover, the modulus of the TBR PU/GE nanofiber membrane was larger than that of the pristine PET fiber braided tube while the elongation at break was lower than that of the PET fiber braided tube. This was because the outer PU/GE nanofiber membrane impeded the fiber slipping and deformation in the PET braided tube during axial stretching, resulting in a decrease in the elongation at break.

\subsection{Wetting properties}

As shown in Fig. 10, the TBR PU/GE nanofiber membranes showed a drastically different response towards water versus oil (kerosene was used as the oil), the water contact angle and the oil contact angle of the PU/GE4-0 nanofiber membrane were $119.2^{\circ}$ and $0^{\circ}$, respectively. As the GE content increased, the water contact angle increased first and then decreased, while the oil contact angle showed no change, when the oil droplet contacted the membrane surface it penetrated into the membrane quickly. This may be ascribed to the contribution of the hydrophobicity of GE. Moreover, the conductivity of the PU/ GE doping solution was enhanced, resulting in a tree-like structure on the nanofiber membrane, and the surface roughness further increases. When the GE content was $0.3 \mathrm{wt} \%$, the water contact angle of the TBR PU/GE nanofiber membrane reached the maximum value of $131.1^{\circ}$. When the GE content continued to increase, the water contact angle decreased owing to GE agglomeration.

\subsection{Pure oil flux}

The results of the pure oil flux of the TBR PU/GE nanofiber membrane with different GE contents are shown in Fig. 11. The pure oil flux of these nanofiber membranes was relatively large,

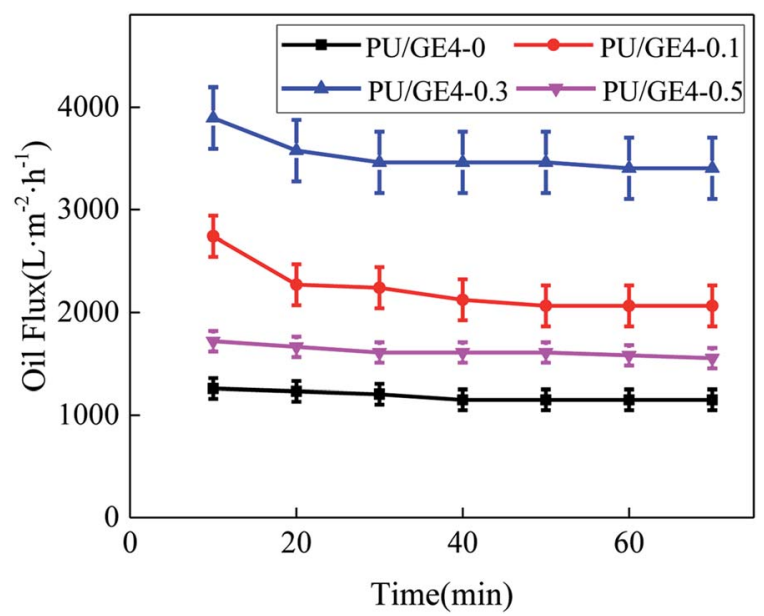

Fig. 11 The oil flux of the PU/GE nanofiber membrane. 

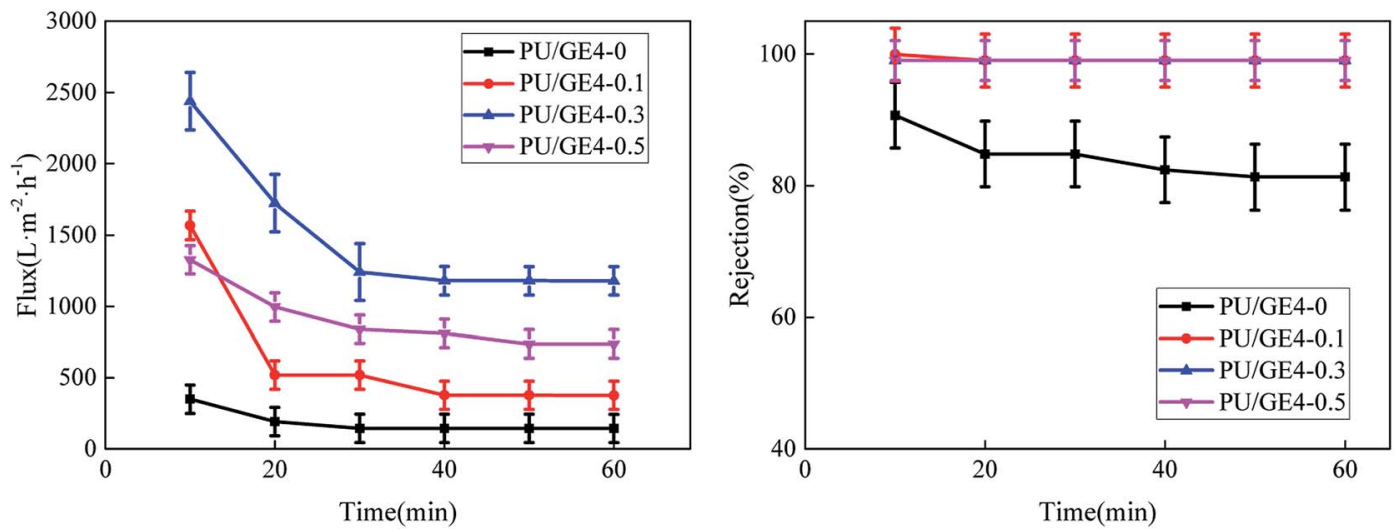

Fig. 12 Permeation flux and rejection of oil/water mixtures.

and the change in the trend for each sample was similar. With the extension of the running time, the pure oil flux of the nanofiber membrane showed a certain decline, and then a flat tendency was reached. Obviously, the pure oil flux of the PU/ GE4-0 nanofiber membrane was smaller than that of the PU/ GE nanofiber membrane. As the GE content increased, the pure oil flux of the PU/GE nanofiber membrane increased first and then decreased. Moreover, GE can improve the hydrophobicity and lipophilicity.

\subsection{Oil-water separation performance test}

To develop the practical applications of the TBR PU/GE nanofiber membrane, the oil/water separation capabilities of the asprepared membranes were studied using a series of oil/water separation processes. An immiscible kerosene and water mixture $(1: 1, \mathrm{v} / \mathrm{v})$ or a water-in-oil emulsion was poured into the glass cylinder as an oil-water mixture, and then the membrane module was placed into the mixture and connected with a negative pressure dead end. Once started, the kerosene

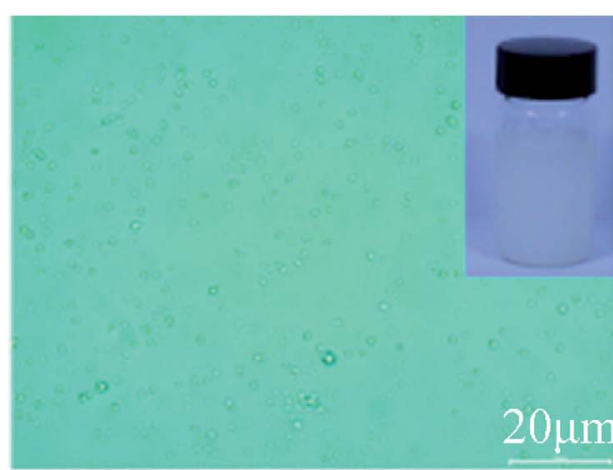

A

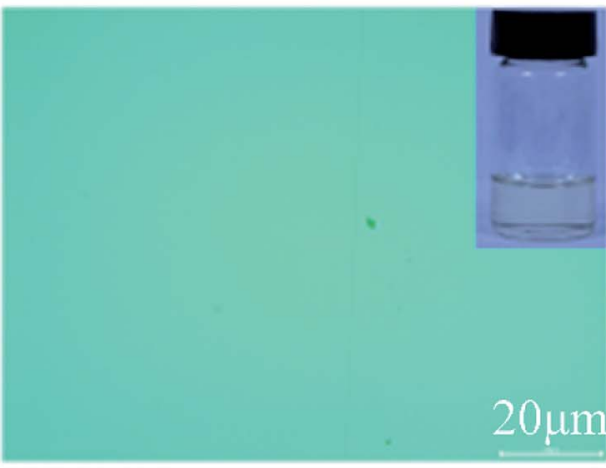

$\mathrm{C}$

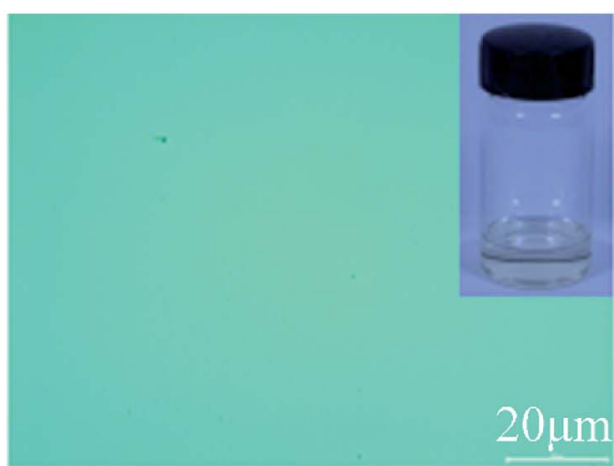

B

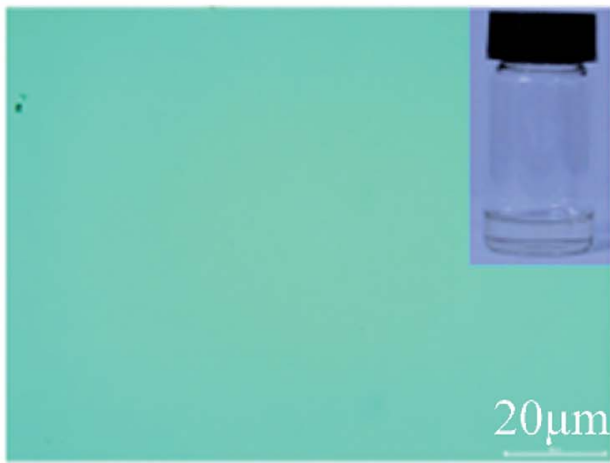

D

Fig. 13 Optical microscopy images of the emulsions before and after filtration: (A) water-in-kerosene emulsion; (B) PU/GE4-0.1; (C) PU/GE40.3; and (D) PU/GE4-0.5. 

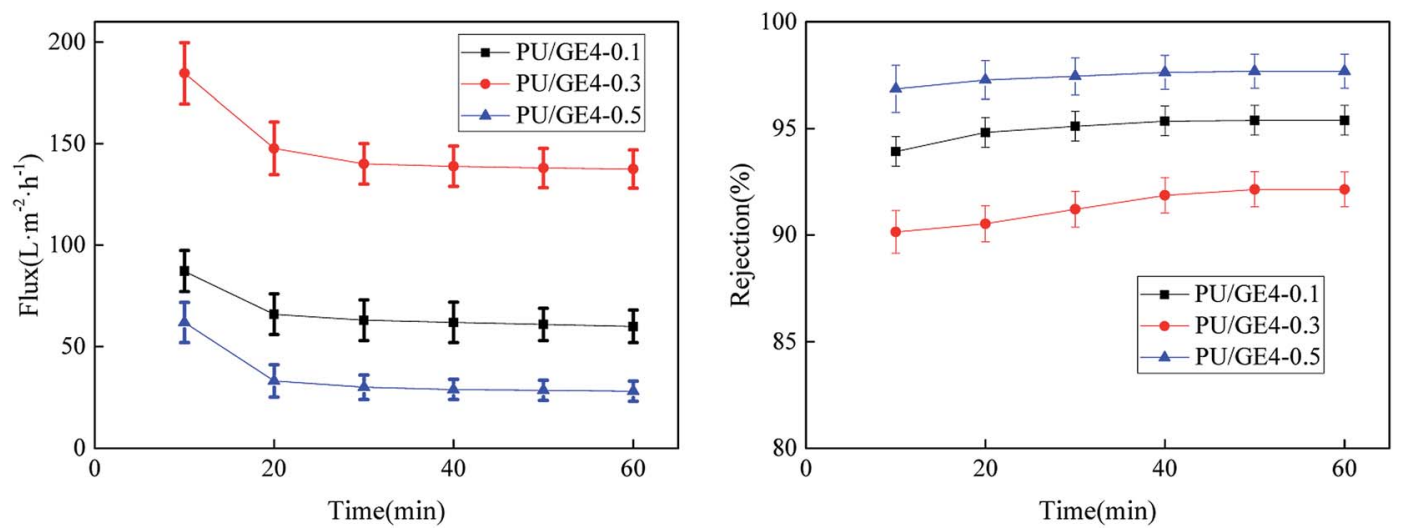

Fig. 14 Permeation flux and rejection of the surfactant-stabilized water-in-oil emulsion.

Table 3 Comparison of the membrane performances obtained in this study with the previously reported oil/water separation processes

\begin{tabular}{lllll}
\hline Reference & $\begin{array}{l}\text { Permeation flux of oil/water } \\
\text { mixtures }\left(\mathrm{L} \mathrm{m}^{-2} \mathrm{~h}^{-1}\right)\end{array}$ & Separation efficiency (\%) & $\begin{array}{l}\text { Permeation flux of water } \\
\text { in oil emulsions }\left(\mathrm{L} \mathrm{m}^{-2} \mathrm{~h}^{-1}\right)\end{array}$ & Separation efficiency $(\%)$ \\
\hline 31 & - & - & 117.3 & - \\
32 & 1136 & 99.0 & - & 92.7 \\
29 & 1333 & 99.9 & 134 & 99.9 \\
This work & 1443 & 99.0 & 137.5 & 90.0
\end{tabular}

quickly penetrated into the membrane, and finally oil was collected in a container. Fig. 12 shows the permeation and separation properties of the TBR PU/GE nanofiber membranes for oil/water separation. The permeation flux of the nanofiber membrane was smaller than the pure oil flux, however, the change in trend was similar. During the oil/water separation process, the oil permeation flux of the PU/GE4-0 nanofiber membrane was $144.3 \mathrm{~L} \mathrm{~m}^{-2} \mathrm{~h}^{-1}$. With the increased GE content, the permeation flux increased first and then decreased. This result was obtained using the pore size and porosity caused by the increasing GE content. For the PU/GE4-0 nanofiber membrane, a certain amount of water can permeate through the PU nanofiber membrane during the filtration process, which makes the separation efficiency low. With the addition of GE, the hydrophobicity of the PU/GE nanofiber membranes increased, leading to a high oil-water separation efficiency, reaching $99 \%$.

For the water-in-oil emulsion separation, once the emulsion came into contact with the PU/GE nanofiber membrane, the emulsion droplets were broken onto the interface of the nanofiber membrane and the oil penetrated the nanofiber membrane and entered the collector. An optical microscope was used to check the separation effect by comparing the water-inkerosene emulsion and the collected filtrate. Fig. 13 shows optical photographs of the water-in-kerosene emulsions and the collected filtrate. As can be seen from the figure, there are many water droplets in the emulsion, but almost no droplets can be observed in the filtrate, indicating that the filtrate was a homogeneous solution with a single component and demonstrating the excellent separating properties of the TBR PU/GE nanofiber membrane. For the separation process of the water-in-kerosene emulsion, the permeation flux and separation efficiency of the TBR PU/GE nanofiber membrane are displayed in Fig. 14. The permeation flux of the water-in-kerosene emulsion was less than that of the oil/water mixture. As a result of the presence of surfactants, the emulsion droplet size was much smaller, which may block the pores of the membrane and result in a reduction in the oil flux. A small amount of water permeation occurred during the oil/water separation process of the pristine PU/GE40 nanofiber membrane, therefore it cannot be used for the separation of the water-in-kerosene emulsion. PU/GE4-0.1, PU/ GE4-0.3, and PU/GE4-0.5 nanofiber membranes showed a good separation performance and the water content in the filtrates of the emulsions was very small. With the increased GE content, the oil permeate flux increased first and then decreased, but the separation efficiency showed the opposite trend. When the GE content was $0.3 \mathrm{wt} \%$, the permeation flux of the TBR PU/GE nanofiber membrane was up to $137.5 \mathrm{~L} \mathrm{~m}^{-2} \mathrm{~h}^{-1}$, and the separation efficiency was more than $90 \%$.

Furthermore, the oil/water separation performances of the obtained membrane were compared with previously reported polymer membranes as summarized in Table 3. It was found that the permeate flux of the PU/GE hollow fiber membrane was higher than that of the other membranes. However, the separation efficiency of our membrane was comparatively lower than the others.

\section{Conclusion}

In summary, a TBR PU/GE nanofiber membrane was prepared using a one-step electrospinning method, in which a PU/GE nanofiber layer was coated onto the surface of a PET braided 
tube. The TBR PU/GE nanofiber membrane showed a good hollow tubular structure and mechanical properties. Moreover, GE and SA assisted the dispersion into the casting solution, improved the hydrophobicity and lipophilicity of the TBR PU/ GE nanofiber membrane and optimized the membrane pore structure. The TBR PU/GE nanofiber membrane could separate the oil-water mixture and the surfactant-stabilized water-in-oil emulsion. The results showed that the TBR PU/GE nanofiber membrane exhibits a stable performance, and the oil-water separation flux was as high as $137.5 \mathrm{~L} \mathrm{~m}^{-2} \mathrm{~h}^{-1}$ and the separation efficiency was $90 \%$. The high porosity, remarkable recyclability and flexibility of the membrane mean it has potential application prospects in industrial applications and environmental protection.

\section{Conflicts of interest}

There are no conflicts to declare.

\section{Acknowledgements}

The authors gratefully acknowledge research funding provided by the National Natural Science Foundation of China (51673149, 51603146), and the Industrial Chain Collaborative Innovation Major Projects of the State Oceanic Administration (BHSF2017-01).

\section{References}

1 C. H. Peterson, S. D. Rice, J. W. Short, D. Esler, J. L. Bodkin, B. E. Ballachey and D. B. Irons, Science, 2003, 302, 20822086.

2 J. Aurell and B. K. Gullett, Environ. Sci. Technol., 2010, 44, 9431-9437.

3 X. Huang, W. Wang, Y. Liu, et al., Chem. Eng. J., 2015, 273, 421-429.

4 T. Dong, S. Cao and G. Xu, J. Hazard. Mater., 2017, 321, 859867.

5 F. Zhang, W. B. Zhang, Z. Shi, D. Wang, J. Jin and L. Jiang, Adv. Mater., 2013, 25, 4192-4198.

6 A. Lee, J. W. Elam and S. B. Darling, Environ. Sci.: Water Res. Technol., 2016, 2, 17-42.

7 A. K. Kota, Y. Li, J. M. Mabry and A. Tuteja, Adv. Mater., 2012, 24, 5838-5843.

8 D. Ishii and M. Shimomura, Chem. Mater., 2013, 25, 509-513.

9 X. Tang, Y. Si, J. Ge, B. Ding, L. Liu, G. Zheng, W. Luo and J. Yu, Nanoscale, 2013, 5, 11657-11664.

10 J. Jeevahan, M. Chandrasekaran, G. B. Joseph, G. M. Durairaj and G. Mageshwaran, J. Coat. Technol. Res., 2018, 15, 231250.
11 V. Pillay, C. Dott, Y. E. Choonara, et al., J. Nanomater., 2013, 2013, 1-22.

12 R. Gopal, S. Kaur, C. Y. Feng, et al., J. Membr. Sci., 2007, 289, 210-219.

13 R. Gopal, S. Kaur, Z. Ma, et al., J. Membr. Sci., 2006, 281, 581586.

14 P. S. Suja, C. R. Reshmi, P. Sagitha and A. Sujith, Polym. Rev., 2017, 57, 467-504.

15 P. Li, S. S. Lim, J. G. Neo, R. C. Ong, M. Weber, C. Staudt, N. Widjojo, C. Maletzko and T. S. Chung, Ind. Eng. Chem. Res., 2014, 53, 14056-14064.

16 C. Zhang, L. Pei and B. Cao, Ind. Eng. Chem. Res., 2015, 54, 8772-8781.

17 S. S. Homaeigohar and M. Elbahri, J. Colloid Interface Sci., 2012, 372, 6-15.

18 P. Arribas, M. Khayet, M. C. García-Payo and L. Gil, Sep. Purif. Technol., 2014, 138, 118-129.

19 T. He, W. Zhou, A. Bahi, H. Yang and F. Ko, Chem. Eng. J., 2014, 252, 327-336.

20 H. R. Pant, H. J. Kim, M. K. Joshi, et al., J. Hazard. Mater., 2014, 264, 25-33.

21 A. M. Bazargan, M. Keyanpour-rad, F. A. Hesari and M. E. Ganji, Desalination, 2011, 265, 148-152.

22 J. J. Li, L. T. Zhu and Z. H. Luo, Chem. Eng. J., 2016, 287, 474481.

23 T. Aslan, S. Arslan, M. Eyvaz, S. Güçlü, E. Yüksel and I. Koyuncu, J. Membr. Sci., 2016, 520, 616-629.

24 C. Su, C. Lu, H. Cao, F. Gao, J. Chang, Y. Li and C. He, Mater. Lett., 2017, 204, 8-11.

25 N. Peng, N. Widjojo, P. Sukitpaneenit, M. M. Teoh, G. G. Lipscomb, T. S. Chung and J. Y. Lai, Prog. Polym. Sci., 2012, 37, 1401-1424.

26 K. C. Khulbe and T. Matsuura, Sep. Purif. Technol., 2016, 2016, 7-13.

27 L.-F. Han, Z.-L. Xu, Y. Cao, Y.-M. Wei and H.-T. Xu, J. Membr. Sci., 2011, 372, 154-164.

28 X. Li, Y. Wang, X. Lu and C. Xiao, J. Membr. Sci., 2008, 320, 477-482.

29 Y. Huang, C. Xiao, Q. Huang, H. Liu, Z. Guo and K. Sun, J. Membr. Sci., 2018, 568, 87-96.

30 X. Qin, L. Jia, W. Lu, D. Shou and J. Fan, Text. Res. J., 2011, 81, 388-397.

31 Y. M. Lin and G. C. Rutledge, J. Membr. Sci., 2018, 563, 247258.

32 W. Ma, Q. Zhang, S. K. Samal, et al., RSC Adv., 2016, 6, 4186141870. 